\title{
A influência de variáveis contextuais e situacionais na classificação de árbitros de futebol de elite.
}

\section{The influence of contextual and situational variables on the classification of elite soccer referees.}

\section{La influencia de las variables contextuales y situacionales en la clasificación de los árbitros de fútbol de élite.}

\author{
Carvalho, V. ${ }^{1}$, Esteves, P.T. ${ }^{2,3}$, Nunes, $C .{ }^{4}$, Mendez, $C .{ }^{5}$, Travassos, B. ${ }^{1,3,6}$ \\ ${ }^{I}$ Department of Sport Sciences, Universidade da Beira Interior, Covilhã, Portugal \\ ${ }^{2}$ Polytechnic Institute of Guarda, Portugal \\ ${ }^{3}$ Research Center in Sports Sciences, Health Sciences and Human Development, CIDESD, Portugal \\ ${ }^{4}$ Department of Mathematics and Center of Mathematics and Applications, \\ Universidade Da Beira Interior, Covilhã, Portugal \\ ${ }^{5}$ Universidad Rey Juan Carlos, Madrid; Universidad Computense de Madrid, Madrid \\ ${ }^{6}$ Portugal Football School, Portuguese Football Federation, FPF, Oeiras, Portugal
}

\section{RESUMO}

Objetivo: Este estudo teve como objetivo avaliar a relação entre a classificação de árbitros de futebol de elite e variáveis contextuais e situacionais que caraterizam os jogos arbitrados no decorrer de uma época. Para tal, foi realizada uma regressão ordinal com função Link Logit entre a classificação final e variáveis contextuais e situacionais. As variáveis contextuais revelaram um efeito significativo sobre a classificação final, não se verificando efeitos significativos das variáveis situacionais, sobre a classificação dos árbitros no final da época desportiva. Na globalidade o modelo revelou-se estatisticamente significativo. A probabilidade de obtenção de melhor classificação final dos árbitros aumenta $54.2 \%$ com o aumento do número de jogos realizados na I Liga e aumenta $24.8 \%$ com aumento do número de jogos equilibrados. Diminui $61.2 \%$ com jogos realizados sem equipas Top 3. Os resultados reforçam a influência significativa que os fatores contextuais têm sobre a classificação e avaliação de um árbitro no final da época desportiva.

Palavras-chave: futebol; arbitragem; desempenho; avaliação; classificação. 


\title{
Carvalho, V., Esteves, P.T., Nunes, C., Mendez, C., Travassos, B.
}

\begin{abstract}
Objective: This study aimed to evaluate the relationship between the classification of elite soccer referees and the contextual and situational variables that characterize the matches refereed during a sports season. So, an ordinal regression with Link Logit function was carried out between the final classification and the contextual and situational variables. The contextual variables revealed a significant effect on the final classification, with no significant effects of the situational variables, on the classification of referees at the end of the sports season. In general, the model proved to be statistically significant. The probability of obtaining a better final referees classification increases $54.2 \%$ with the increase in the number of games played in the I League and $24.8 \%$ with the increase in the number of balanced games. Decreases $61.2 \%$ with games played without Top 3 teams. The results reinforce the significant influence that contextual factors have on the classification and assessment of a referee at the end of the sports season. Keywords: soccer; refereeing; performance; evaluation; classification.
\end{abstract}

\section{RESUMEN}

Objetivo: Este trabajo tuvo como objetivo evaluar la relación entre la clasificación de árbitros de fútbol de élite y las variables contextuales y situacionales que caracterizan los juegos de árbitrados durante la temporada desportiva. Para ello se realizó una regresión ordinal con función Link Logit entre la clasificación final y las variables contextuales y situacionales. Las variables contextuales tuvieron un efecto significativo en la clasificación final, sin efectos significativos de las variables situacionales, en la clasificación de los árbitros al final de la temporada deportiva. En general, el modelo se ai revelado como estadísticamente significativo. La probabilidad de obtener una mejor clasificación final de los árbitros aumenta 54,2\% con el incremento del número de partidos julgados en La Liga, y $24,8 \%$ con el incremento del número de partidos equilibrados. Disminuye $61,2 \%$ con partidos jugados sin equipos Top 3. Los resultados refuerzan la influencia significativa que los factores contextuales tienen en la clasificación y evaluación de un árbitro al final de la temporada deportiva.

Palabras clave: fútbol; arbitraje; rendimiento; evaluación; clasificación.

\section{INTRODUÇÃO}

Fruto da grande variabilidade e dinâmica situacional que caraterizam o jogo de futebol, o árbitro de futebol de elite necessita realizar de forma continua decisões que lhe permitam, no decorrer do jogo, assegurar o cumprimento das regras do jogo (Elsworthy, Burke, Scott, Stevens, \& Dascombe, 2014). Ao longo dos últimos anos, muitos estudos avaliaram a prestação do árbitro de futebol tendo por base a avaliação da tomada de decisão do árbitro (Elsworthy et al., 2014). Esta foi aliás, durante largos anos considerada a competência mais importante do árbitro (Helsen \& Bultynck, 2004; Morris \& O'Connor, 2016), sendo avaliada como um meio eficaz para diferenciar peritos e não peritos (Berry, Abernethy, \& Côté, 2008; Williams \& Ericsson, 2005), pois, considera-se que reflete o nível de adaptabilidade do árbitro à variabilidade do próprio jogo (Urra, Nuñez, Oses, \& Sarmiento, 2018; Kittela, Larkina, Elsworthy, \& Spittle, 2019)._No entanto, esta tarefa não tem apenas por base o cumprimento estrito das regras do jogo (Guillén \& Jiménez, 2001), sendo influenciada pela avaliação constante dos seus pares e do público em geral (Collina, 2003), necessitando o árbitro para isso de grande resiliência e motivação para o desempenho das suas funções (Folkesson, Nyberg, Archer, \& Norlander, 2002; Wolfson \& Neave, 2007).

A este propósito, Pina, Passos, Carvalho e Maynard (2019) consideram que o desenvolvimento de excelência e das competências do árbitro são altamente influenciadas pelo processo que as medeia, nomeadamente as classificações, promoções e as despromoções de categoria, o apoio psicológico, a pressão a humilhação pública na televisão e nos jornais diários a que estão sujeitos (Weaver, 2001; Wilson, 2000), bem como os programas de formação e de treino existentes. Mascarenhas et al. (2005) identificaram cinco fatores que sustentam a excelência no desempenho da arbitragem: a) competências de personalidade e na gestão do jogo, b) julgamento contextual, c) conhecimento e aplicação da lei, d) características psicológicas de excelência e e) aptidão física, posicionamento e qualidades mecânicas. Estes fatores foram corroborados em outros estudos realçando que a experiência na arbitragem em 
contextos adversos (Catteeuw, Helsen, Gilis, \& Wagemans, 2009), competências na gestão do jogo (Sabatini, 2002) bem como competências de comunicação, de gestão das emoções (Dosseville, Laborde, \& Bernier, 2014), de inteligência emocional, e liderança para além do conhecimento de regulamentos e aspetos técnicos são fundamentais para a excelência na arbitragem (Sülün, 2013). Em suma, a prestação do árbitro de futebol é influenciada por fatores: a) contextuais que marcam o contexto de jogo e o ambiente que o rodeia; b) situacionais que ocorrem durante o jogo, fruto da dinâmica do mesmo e da relação estabelecida entre árbitro, jogadores, treinadores e público; e c) individuais fruto da preparação e capacidade do próprio árbitro para gerir o jogo.

A respeito dos fatores contextuais que influenciam a prestação do árbitro de futebol, Nevill, Balmer e Williams (2002), revelaram que, num estudo experimental, os árbitros que ouviam o barulho do público num clip de vídeo, eram influenciados na decisão sobre possível situação de infração da equipa da casa, do que os árbitros que assistiram aos clipes sem som ambiente. No entanto, embora fatores contextuais (equipa da casa ou público) pareçam influenciar a tomada de decisão do árbitro em competições nacionais (Buraimo, Forrest, \& Simmons, 2010; Buraimo, Simmons, \& Maciaszczyk, 2012), o mesmo não se verificou em competições internacionais com árbitros de elite. De modo semelhante, parece que o nível competitivo das equipas parece influenciar a gestão e preparação do próprio jogo (Tenreiro-Gavela, Montero-Seone, \& Saavedra-García, 2016), bem como o modo como os árbitros decidem a atribuição de um penalti (Erikstad \& Johansen, 2020). Por outro lado, Jones, Paull e Erskine (2002) verificaram que o conhecimento prévio dos árbitros acerca da agressividade das equipas influenciava a exibição de cartões amarelos, isto é, à equipa considerada previamente mais agressiva eram mostrados mais cartões amarelos do que à equipa contrária.

No que diz respeito aos fatores situacionais, o modo como o árbitro realiza a gestão do jogo pode levar a que a mesma tomada de decisão ou uma ação disciplinar possa ser interpretada como um erro de julgamento face às leis de jogo, ou pode ser vista como uma decisão correta de modo a salvaguardar a gestão e fluidez do jogo (Askins, 2001; Grunska, 2001). Por exemplo, Brand et al. (2006) verificaram num estudo experimental que, quando os árbitros viram uma sequência de clipes com infrações pela ordem em que ocorreram durante o jogo, assumiram sanções menos rigorosas e mais diversas, do que ao ver as mesmas como uma infração isolada. Plessner e Betsch (2001), revelaram que a decisão de assinalar um penalti, poderá ser influenciada por decisões anteriores em situações similares, já tendo sido demonstrado em outros contextos desportivos que as "regras não escritas" podem influenciar as decisões dos árbitros (Plessner \& Raab, 1999). De forma semelhante, Lex, Pizzera, Kurtes e Schack (2015) revelaram ainda que a ação disciplinar do árbitro através de cartão amarelo era mais vezes registada perante a vocalização do jogador e pela necessidade de gestão do jogo do que propriamente fruto de uma infração às leis de jogo.

Fruchart e Carton (2012), em relação às caraterísticas individuais, indicaram que os árbitros de nível inferior tendem a cometer mais erros e de alguma forma premeditada, tornando a gestão do mesmo mais difícil. No entanto, tal como referido anteriormente, esta parece ser uma estratégia de valorização individual fruto das caraterísticas que assumem nomeadamente as classificações que dão origem às promoções e despromoções dos árbitros no final da época desportiva.

A respeito da avaliação da prestação do árbitro de futebol e consequentemente da classificação no final da época, Weaver (2001) considera que este processo influencia bastante a prestação do árbitro no decorrer do jogo, não o permitindo em algumas circunstâncias de adotar uma gestão do jogo mais eficaz e de acordo com o contexto em que este ocorre, em detrimento do estrito cumprimento das leis de jogo. As avaliações do desempenho do árbitro, são normalmente efetuadas por ex-árbitros experientes, que de acordo com as diretrizes da FIFA, UEFA e do próprio conselho de arbitragem da federação nacional avaliam e classificam o desempenho do árbitro de futebol, num determinado jogo. Esta avaliação é realizada tendo por base uma observação ao vivo ou em vídeo tendo por base grelhas de observação que consideram as seguintes categorias de observação: i) dificuldade do jogo e intervenção, no qual se tem em consideração fatores contextuais, capacidade individual e capacidade de posicionamento e colaboração entre membros da equipa de arbitragem; ii) ações disciplinares e gestão do jogo, no qual se consideram aspetos relacionados com ação disciplinar, aplicação das leis de jogo e controlo disciplinar. 


\section{Variáveis contextuais e situacionais na classificação de árbitros de futebol de elite}

Sabendo da pertinência e influência destes processos de observação na prestação imediata dos árbitros, bem como no desenvolvimento da sua carreira desportiva, nada se sabe, no entanto, sobre a possível influência de algumas condições contextuais e situacionais na avaliação do desempenho do árbitro e consequente da sua classificação no final da época. De facto, não é conhecido até ao momento nenhum estudo que tenha verificado a influência de fatores contextuais ou situacionais na classificação obtida no jogo, bem como na respetiva classificação final dos árbitros no final de cada temporada.

Deste modo, este estudo teve como objetivo, analisar a relação entre a classificação dos árbitros de futebol de elite em Portugal e as variáveis contextuais e situacionais dos jogos arbitrados no decorrer da época desportiva 2016-2017. Como hipóteses consideramos que: i) a classificação dos árbitros de futebol é influenciada por variáveis contextuais (Sülün, 2013). Os árbitros melhor classificados tendem a arbitrar jogos de competições de nível superior, entre as melhores equipas, e jogos mais equilibrados em comparação com árbitros pior classificados; ii) a classificação dos árbitros de futebol é influenciada pelo número de ações disciplinares no decorrer do jogo (Fruchart \& Carton, 2012). Os árbitros melhor classificados tendem a apresentar menor número de ações disciplinares em comparação com árbitros pior classificados.

\section{MÉTODOS}

\section{Desenho experimental}

Este estudo teve como base um desenho empírico de natureza associativa que envolveu a comparação de variáveis experimentais entre diferentes grupos com o intuito de explorar associações de funcionalidade e eventuais diferenças (Ato, López, \& Benavente, 2013).

\section{Participantes}

Vinte e três árbitros de futebol (média de idades $=36,6 \pm 4,08$ anos) que constituíam o quadro de árbitros da $1^{a}$ categoria em Portugal na época 20162017 foram considerados para o estudo. Os últimos quatro classificados desciam de categoria. A cada árbitro foi atribuído um Código e registados quinhentos e cinquenta e oito jogos no âmbito da I Liga e II Liga. A cada árbitro foi ainda atribuída a respetiva classificação final oficial da época, tendo por base o somatório da avaliação de cada jogo pela Federação Portuguesa de Futebol. Este trabalho foi aprovado pelo comité de ética da Universidade da Beira Interior de acordo com a declaração de Helsínquia (2013).

\section{Recolha de dados}

Todos os dados foram selecionados e recolhidos da base de dados digital privada zerozero (www.zerozero.pt) e registados numa matriz de excel. Como variável dependente foi considerada a variável classificação final dos árbitros na época 2016-2017, através da divisão da classificação em tercis: $1^{\circ}$ tercil (G1) - classificação superior (do $1^{\circ}$ ao $8^{\circ}$ classificado inclusive); $2^{\circ}$ tercil (G2) - classificação intermédia (do $9^{\circ}$ ao $16^{\circ}$ classificado ambos inclusive); $3^{\circ}$ tercil (G3) - classificação inferior (do $17^{\circ}$ ao $23^{\circ}$ classificado ambos inclusive).

Como variáveis independentes foram definidas variáveis contextuais: i) o nível da competição (1 - I Liga; 2 - II Liga), ii) Caraterização nível competitivo do jogo (Outros x Outros: jogos sem equipas do top 3 do campeonato; Top 3 x Outros: jogos com uma equipa Top 3 e outra equipa que não Top 3 do campeonato; Top 3 x Top 3: jogos entre equipas do Top 3 do campeonato) (A caraterização do nível competitivo do jogo foi considerada em três níveis tendo por base os critérios de avaliação de desempenho do árbitro de futebol em vigor: jogo com participação de pelo menos uma equipa Top 3 é considerado como um jogo de dificuldade mais elevado; jogo com participação de duas equipas Top 3 é considerado jogo de dificuldade superior; jogo sem participação de equipas Top 3 é considerado como um jogo de dificuldade normal); iii) resultado do jogo (1 - equilibrado - quando existe uma diferença até dois golos; 2 - empate, 3 - desequilibrado - quando existe uma diferença superior a dois golos); e variáveis situacionais relacionadas com a ação disciplinar do árbitro no decorrer do jogo: iv) número total de cartões exibidos por jogo (total de cartões amarelos e vermelhos exibidos por jogo).

\section{Análise estatística}

No sentido de analisar qual a relação entre a classificação final e o nível da competição, caraterização do nível competitivo do jogo, resultado do jogo e número total de cartões exibidos por jogo 
foi realizada uma regressão ordinal com função Link Logit. A seleção da função Link foi baseada na frequência de distribuição das classes das variáveis dependentes (Marôco, 2014). O pressuposto do modelo da homogeneidade de declives foi validado $\left(X_{\mathrm{LP}}^{2}(5)=3.356, \mathrm{p}=0.645\right)$. Os odds ratios $(\mathrm{OR})$ foram estimados para as classificações superiores, em comparação com as classificações inferiores. A análise estatística foi efetuada com recurso ao SPSS v 23.0 para MAC (IBM Corp.). Foi considerada uma significância estatística de 5\%.

\section{RESULTADOS}

Através da análise da Tabela 1, verificámos que, em geral, para todas as variáveis contextuais, os valores de G1 são mais elevados em comparação com o G2 e G3. De forma mais específica, os árbitros G1 realizaram mais jogos de nível de competição superior (I Liga), realizaram mais jogos com equipas Top $3 \mathrm{e}$ apresentaram valores médios em termos de resultado de jogo quando comparados com o G2 e G3. Em relação às variáveis situacionais, os árbitros do G1 foram os que apresentaram menor número de cartões exibidos por jogo. Os resultados alcançados pelo G2, estão tendencialmente na mediana, entre o G1 e G3 (Tabela 1).

\begin{tabular}{|c|c|c|c|c|}
\hline Variáveis & & G1 & G2 & G3 \\
\hline \multicolumn{5}{|c|}{ Variáveis contextuais } \\
\hline \multirow{2}{*}{ Nivel da competição } & I Liga & $63,33 \%$ & $52,38 \%$ & $42,14 \%$ \\
\hline & II Liga & $36,67 \%$ & $47,62 \%$ & $57,86 \%$ \\
\hline \multirow{2}{*}{ Caracterização do jogo } & Outros & $73,81 \%$ & $83,07 \%$ & $93,71 \%$ \\
\hline & TOP 3 & $26,19 \%$ & $16,93 \%$ & $6,29 \%$ \\
\hline \multirow{3}{*}{ Resultado do jogo } & Equilibrado & $56,67 \%$ & $61,38 \%$ & $67,92 \%$ \\
\hline & Empate & $32,86 \%$ & $29,10 \%$ & $21,38 \%$ \\
\hline & Desequilibrado & $10,48 \%$ & $9,52 \%$ & $10,69 \%$ \\
\hline \multicolumn{5}{|c|}{ Variáveis Situacionais } \\
\hline $\begin{array}{l}\text { Número total de cart } \\
\text { exibidos por jogo }\end{array}$ & & $37,76 \%$ & $33,71 \%$ & $28,53 \%$ \\
\hline
\end{tabular}

Os resultados demonstraram que, na globalidade, o modelo se revelou estatisticamente significativo $\left(X_{\mathrm{LP}}^{2}(5)=40.299, \mathrm{p}<0.001\right)$. Os jogos realizados na I Liga aumentam a chance de obtenção de melhor classificação final dos árbitros em 54.2\% quando comparados com os jogos realizados na II Liga $(\mathrm{p}=0.011 ; \quad O R=1.542 ;$ IC $95 \%=[1.102 ; 2.154])$. Os jogos realizados sem equipas Top 3 diminuem em $61.2 \%$ a possibilidade dos árbitros obterem melhor classificação quando comparados com os jogos realizados com equipas Top 3 ( $\mathrm{p}<0.001 ; O R=0.388$; IC $95 \%=[0.241 ; 0.624])$.

Um resultado equilibrado do jogo aumenta em $24.8 \%$ a possibilidade de obtenção de uma melhor classificação, apesar de não significativa, $(\mathrm{p}=0.425$; $\mathrm{OR}=1.248$; IC 95\%=[0.724; 2.148$]$ ), enquanto que um empate duplica a chance de uma melhor classificação final quando comparado com um resultado desequilibrado do jogo ( $\mathrm{p}=0.019 ; \quad O R=2.033$; IC $95 \%=[1.125 ; 2.148])$. Por último, em relação ao número total de cartões exibidos num jogo, não se verificaram efeitos significativos no ranking de desempenho dos árbitros $(\mathrm{p}=0.566 ;$ OR=1.020; IC $95 \%=[0.952 ; 1.094])$.

\section{DISCUSSÃO}

O principal objetivo deste estudo foi avaliar qual a relação entre as variáveis contextuais e situacionais em estudo e a classificação dos árbitros de futebol de elite em Portugal, no decorrer da época desportiva de 2016-2017. De acordo com as hipóteses consideradas, os resultados evidenciaram, que a combinação entre as variáveis contextuais e situacionais exploradas, por si só, explicam cerca de $40 \%$ da classificação final dos árbitros de futebol de elite analisados. Especificamente, as variáveis contextuais, apresentam um efeito significativo sobre a classificação final dos árbitros de futebol no final da época desportiva. Ao invés do esperado, não se verificaram efeitos significativos das variáveis situacionais, número de ações disciplinares, sobre a classificação final dos árbitros de futebol no final da época desportiva.

Qualquer processo de avaliação de desempenho, e em particular na arbitragem, fruto do peso e influência que tem sobre os próprios árbitros (Pina et al., 2019), deve ter como objetivo a avaliação dos fatores que sustentam a excelência na arbitragem, bem como a identificação de medidas tendentes ao aperfeiçoamento dessa intervenção. Sendo este um processo que para além do aspeto classificativo, tem implicações nas promoções e despromoções de categoria, devemos procurar a melhor compreensão sobre o instrumento utilizado, bem como do peso de cada fator para o processo de classificação final (Mascarenhas, Collins, \& Mortimer, 2005). A este propósito podemos referir que os observadores de 


\section{Variáveis contextuais e situacionais na classificação de árbitros de futebol de elite}

árbitros assumem uma responsabilidade e uma exigência na sua atuação crucial para a classificação dos árbitros de futebol e seu consequente desenvolvimento de carreira (Fruchart \& Carton, 2012; Mathers \& Brodie, 2011).

Tendo por base as exigências da atividade de arbitragem, mas também o contexto em que estas se desenvolvem, Mascarenhas et al. (2005) verificaram que a performance do árbitro resulta da sua capacidade individual para intervir e gerir os fatores contextuais que marcam o contexto de jogo e o ambiente que o rodeia, e os fatores situacionais determinados pela dinâmica do jogo e aspetos disciplinares que o constituem. Não sendo possível estudar, em articulação, a avaliação entre estes 3 fatores e o modo como concorrem para a avaliação final do árbitro de futebol, é possível avaliar o modo como os fatores contextuais que caraterizam os jogos e o ambiente que os rodeia e os fatores situacionais definidos pelo carater disciplinar da ação do árbitro na sua avaliação final.

Como referido anteriormente, os resultados obtidos revelaram que os fatores contextuais apresentaram valores percentuais mais elevados para o grupo dos árbitros melhor classificados em comparação com os pior classificados, nomeadamente no que diz respeito ao número de jogos realizados por equipas da I Liga, aos jogos em que participaram os três primeiros classificados do campeonato e aos jogos que terminaram em empate. Já os árbitros que terminaram pior classificados apresentaram maior percentagem de jogos realizados por equipas da II Liga, jogos em que participaram outras equipas que não as três primeiras classificadas, e jogos desequilibrados.

De um modo geral considera-se que a atuação do árbitro, bem como a sua tomada de decisão pode ser afetada pelo contexto de jogo, nomeadamente pela avaliação do público e comunicação social, treinadores, dirigentes e jogadores (Collina, 2003). No entanto, de acordo com os resultados apresentados, os melhores árbitros parecem não sofrer tanto esta pressão do contexto do jogo, e possíveis consequências da avaliação do público e comunicação social sobre a sua atuação ou imagem (Catteeuw et al. 2009; Buraimo et al. 2012). Sabendo que os melhores tendem a arbitrar os jogos de contextos tendencialmente mais difíceis, é coerente que o processo de avaliação reflita essa mesma capacidade ao permitir que estes arbitrem mais jogos da I Liga, onde participam as equipas de maior impacto social (três primeiros classificados) e os jogos mais equilibrados. No entanto, face ao peso significativo que estas variáveis apresentam para a classificação final (cerca de 40\%), consideramos que a classificação final pode ser enviesada logo à partida pelas nomeações realizadas para estes jogos. Nomeadamente porque de acordo com os critérios de avaliação de desempenho do árbitro de futebol em vigor, um jogo com uma das equipas Top 3 é considerado automaticamente como um jogo de dificuldade mais elevado, aumentando de imediato a classificação final do árbitro em comparação com um árbitro que tenha o mesmo desempenho, mas num jogo entre outros ou entre equipas da II Liga.

De forma interessante, constatamos que dos quatro últimos árbitros na classificação final, e que desceram de categoria, apenas o último classificado foi nomeado para mais jogos da I Liga (12) do que da II Liga (10). Do mesmo modo, o último classificado foi nomeado para dois jogos com a participação de equipas Top 3, enquanto os restantes três árbitros não foram nomeados para nenhum jogo Top 3. Estes resultados realçam que, apesar da tendência verificada ao nível das variáveis contextuais, existem casos em que o peso das variáveis relacionados com a ação mais técnica do árbitro (intervenção, gestão do jogo, aplicação das leis de jogo) poderão ter um peso significativo na sua classificação, bem como a articulação entre ambas é evidente. Por exemplo, um fraco desempenho num jogo terá reflexos diretos na classificação final do árbitro, bem como indiretamente terá repercussões sobre o nível de jogos em futuras nomeações, o que se poderá aparentemente tornar um fator negativo, para a sua classificação final.

Estudo futuros são necessários para avaliar o peso de cada uma das variáveis do relatório de avaliação, na classificação final do jogo, bem como da época desportiva de um árbitro de futebol. Dessa forma será possível identificar com maior precisão qual a influência destas variáveis contextuais na avaliação da prestação de cada árbitro de futebol.

Por sua vez, estudos anteriores têm revelado que a gestão dos aspetos disciplinares por árbitros peritos, em comparação com árbitros de menor experiência, nomeadamente a amostragem de cartões amarelos ou vermelhos (Fruchart \& Carton, 2012) poderão ser influenciadas por "regras não escritas" (Plessner \& Raab, 1999) permitindo uma gestão do jogo mais eficaz e de acordo com o contexto em que este ocorre 
(Askins, 2001; Grunska, 2001), em detrimento do estrito cumprimento das leis de jogo (Weaver, 2001). No entanto, os resultados obtidos não nos revelaram qualquer efeito ao nível do número de cartões amarelos e vermelhos mostrados por jogo. A este propósito, provavelmente, mais do que o número de cartões, a classificação final será mais influenciada pela avaliação da intervenção disciplinar do que pela quantidade de intervenções em si mesma. Por outro lado, ao invés dos árbitros de nível inferior, que tendem a mostrar mais cartões amarelos, aumentando o grau de dificuldade do jogo e consequentemente a classificação no mesmo em termos de dificuldade de gestão do jogo (Fruchart \& Carton, 2012), os árbitros analisados constituíam o quadro de árbitros da $1^{\text {a }}$ categoria, sendo todos considerados como árbitros peritos, e a arbitrar as Ligas mais competitivas e mediáticas em Portugal. De acordo com o referido, estudos futuros deverão considerar mais do que o número de cartões amarelos e vermelhos mostrados, a sua adequabilidade de acordo com a aplicação das leis do jogo, bem como o número de infrações técnicas e ocorrências de registadas no decorrer do jogo (Catteeuw et al., 2009).

Face ao verificado, são necessários estudos futuros no sentido de averiguar qual o peso que cada critério de classificação tem na classificação final do árbitro, de modo a averiguar se existe correspondência com o determinado em termos teóricos para avaliação do desempenho do árbitro de futebol. Como limitação deste estudo apontamos o facto de considerar apenas uma época desportiva. Para uma melhor compreensão do peso, sobretudo das variáveis contextuais, na classificação dos árbitros de futebol, estudo com amostras superiores devem ser considerados de modo a uma maior generalização dos resultados.

\section{IMPLICAÇÕES PRÁTICAS}

Em suma, os resultados alcançados, evidenciaram por um lado que o número de ocorrências relacionadas com fatores situacionais avaliados, por si só, possuem uma influência residual na classificação final do árbitro de futebol, enquanto que os fatores contextuais têm uma influência significativa sobre a classificação e avaliação de um árbitro no final da época desportiva, contribuindo para a subida (ou indicação à FIFA) de categoria do árbitro, a sua manutenção ou para a descida de categoria. No global, apenas considerando as variáveis contextuais e situacionais o modelo explica cerca de $40 \%$ da classificação final dos árbitros de futebol. Em termos práticos, tal significa, que por si só o contexto do número de jogos que arbitra poderá influenciar em $40 \%$ a sua classificação final.

\section{REFERÊNCIAS}

Askins, R. (2001). Common myths about officiating. Referee, 26(10), 44-47.

Ato, M., López, J., Benavente, A. (2013). Un sistema de clasificación de los diseños de investigación en psicología. Anales de Psicología, 29(3), 10381059. doi:10.6018/analesps.29.3.178511

Berry, J., Abernethy, B., Cote, J. (2008). The contribution of structured activity and deliberate play to the development of expert perceptual and decision-making skill. Journal of Sport \& Exercise Psychology, 30(6), 685-708. doi:10.1123/jsep.30.6.685

Brand, R., Schmidt, G., Schneeloch Y. (2006). Sequential effects in elite basketball referees' foul decisions: An experimental study on the concept of game management. Journal of Sport and Exercise Psychology, 28, 93-99. doi:10.1123/jsep.28.1.93

Buraimo, B., Forrest, D., Simmons, R. (2010). The 12th man?: refereeing bias in English and German soccer. Journal of the Royal Statistical Society: Series A (Statistics in Society), 173(2), 431-449. doi:10.1111/j.1467-985X.2009.00604.X

Buraimo, B., Simmons, R., Maciaszczyk, M. (2012). Favoritism and referee bias in european soccer: evidence from the spanish league and the UEFA champions league. Contemporary Economic Policy, 30(3), 329-343. doi:10.1111/j.1465-7287.2011.00295.x

Catteeuw, P., Helsen, W. F., Gilis, B., \& Wagemans, J. (2009). Decision-making skills, role specificity, and deliberate practice in association football refereeing. Journal of Sports Sciences, 27(11), 1125-1136. doi:10.1080/02640410903079179

Collina, P. (2003). The rules of the game. London: Macmillan

Dohmen, T., \& Sauermann, J. (2016). Referee bias. Journal of Economic Surveys, 30(4), 679-695. doi:10.1111/joes.12106 


\section{Variáveis contextuais e situacionais na classificação de árbitros de futebol de elite}

Dosseville, F., Laborde, S., Bernier, M. (2014). Athletes' expectations with regard to officiating competence. European Journal of Sport Science, 14(1), 448-455. doi:10.1080/17461391.2012.713006

Elsworthy, N., Burke, D., Scott, B., Stevens, C., Dascombe, B. (2014). Physical and decisionmaking demands of Australian football umpires during competitive matches. Journal of Strength and Conditioning Research, 28(12), 3502-3507. doi:10.1519/JSC.0000000000000567

Erikstad, M. K., Johansen, B. T. (2020). Referee bias in professional football: Favoritism toward successful teams in potential penalty situations. Frontiers in Sports and Active Living, 2(19). doi:10.3389/fspor.2020.00019

Folkesson, P., Nyberg, C., Archer, T., Norlander, T. (2002). Soccer referees' experience of threat and agression: Effects of age, experience, and life orientation on outcome of coping strategy. Aggressive Behavior, 28, 317-327. doi:10.1002/ab.90028

Fruchart, E., Carton, A. (2012). How do amateur soccer referees destabilize a match? Psicologica: International Journal of Methodology and Experimental Psychology,33(3), 435-449.

Grunska, J. (2001). Yeah, you're good...But you can be better. Referee 26(6), 36-40.

Guillén, F., Jiménez, H. (2001). Características deseables en el arbitraje y el juicio deportivo. Revista De Psicologia Del Deporte, 10(1), 23-34.

Helsen, W., Bultynck, J-B. (2004). Physical and perceptual-cognitive demands of topclass refereeing in association football. Journal of Sports Sciences, 22(2), 179-189. doi:10.1080/02640410310001641502

Jones, M.V., Paull, G.V., Erskine, J. (2002). The impact of team's aggressive reputation on the decisions of association football referees. Journal of Sport Sciences, 20, 991-1000. doi:10.1080/026404102321011751

Kittela, A., Larkina, P., Elsworthy, N., Spittle, M. (2019). Video-based testing in sporting officials: A systematic review. Psychology of Sport \& Exercise, 43, 261-270. doi:10.1016/j.psychsport.2019.03.013

Lex, H., Pizzera, A., Kurtes, M., Schack, T. (2015). Influence of players "vocalisations on soccer referees" decisions. European Journal of Sport Science, 15(5), 24-28. doi:10.1080/17461391.2014.962620

Marôco, J. (2014). Análise de Equações Estruturais. (2nd ed.). Pêro Pinheiro, Report Number. Retrieved from

http://www.reportnumber.pt/aee/

Mascarenhas, D., Collins, D., Mortimer, P. (2005). Elite refereeing performance: Developing a model for sport science support. The Sport Psychologist, 19, 364-379. doi:10.1123/tsp.19.4.364

Mathers, J. F., Brodie, K. (2011). Elite refereeing in professional soccer: A case study of mental skills support. Journal of Sport Psychology in Action, 2(3), 171-182. doi:10.1080/21520704.2011.609018

Morris, G., Connor D. (2016). Key attributes of expert NRL referees. Journal of Sports Sciences, 35(9), 852-857. doi:10.1080/02640414.2016.1194524

Nevill, A. M., Balmer, N. J., Williams, A. M. (2002). The influence of crowd noise and experience upon refereeing decisions in football. Psychology of Sport and Exercise, 3(4), 261-272. doi:10.1016/S1469-0292(01)00033-4

Pina J. A., Passos A. M., Carvalho, H., Maynard M. T. (2019). To be or not to be an excellent football referee: Different experts' viewpoints. Journal of Sports Sciences, 37(6), 692-700. doi:10.1080/02640414.2018.1522940

Plessner, H., Raab, M. (1999). Kampf-und Schiedsdchterurteile als Produkte sozialer Informations verarbeitung [Judgments by officials in sports as products of social information processing]. Psychologie \& Sport, 6, 130-145.

Plessner H., Betsch, T. (2001). Sequential effects in important referee decisions: The case of penalties in soccer. Journal of Sport \& Exercise Psychology, 23, 254-259.

Sabatini, D. (2002). The search for excellence. Referee, 24-28.

Sülün, Ö., (2013). Comparison of anger and furiousness levels of soccer referees with their empathetic tendencies. Master's thesis. Karamanoğlu Mehmet Bey University, Institute of Social Sciences, Karaman.

Tenreiro-Gavela, F., Montero-Seone, A., SaavedraGarcía, M. (2016). La autopercepción del esfuerzo y recuperación en el arbitraje profesional español: Un estudio de caso. 
Cuadernos De Psicología Del Deporte, 16(3), 137-144. doi:10.4321/s1578-84232013000200011

Urra, B., Nuñez, C., Oses, J., Sarmiento, G. (2018). Variables psicológicas influyentes en el desempeño de árbitros FIFA: Un estudio cualitativo. Revista de Psicología Aplicada al Deporte y al Ejercicio Físico, 3(2), 1-11. doi:10.5093/rpadef2018a13

Weaver, P. (2001). No hiding place for Gallagher. The guardian unlimited. Monday November 12th. Electronic article retrieved from http://football.guardian.co.uk/Match Report/0,5 91738,00.html

Williams, M., Ericsson, A. (2005). Perceptualcognitive expertise in sport: Some considerations when applying the expert performance approach. Human Movement Science, 24(3), 283-307. doi:10.1016/j.humov.2005.06.002
Wilson, R. (Producer \& Director) (2000, July 25). Man in black. Scotland: British Broadcasting Corporation.

Wolfson, S., Neave, N. (2007). Coping under pressure: Cognitive strategies for maintaining confidence among soccer referees. Journal of Sport Behavior, 30, 232-247.

\section{Agradecimentos}

Este estudo foi suportado pela Fundação para Ciência e Tecnologia com o número de registo

UID04045/2020 para os autores PTE e BT.

\section{Conflitos de interesse}

Nada a declarar. 